\title{
Propuesta de un plan educativo en mecánica corporal para el personal de enfermería hospitalaria
}

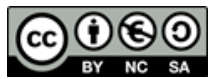

Proposal for an educational plan in body mechanics for hospital nursing staff
\end{abstract}

Gladys Magdalena Naranjo Chávez. ${ }^{1}$, Liliana Raquel Rojas González. ${ }^{2}$, María Humbelina Olalla García. $^{3}$

Recibido: 10-08-2021 / Revisado: 15-08-2021 /Aceptado: 23-09-2021/ Publicado: 05-10-2021

DOI: https://doi.org/10.33262/anatomiadigital.v4i4.1920

\begin{abstract}
Introduction. The practice of hospital nursing requires a high demand for physical effort, which due to ignorance can cause musculoskeletal injuries. Objective, Propose an educational plan in body mechanics for hospital nursing staff. Methodology. Based on a previous descriptive, prospective, cross-sectional research, with a non-experimental design, in a sample of 124 subjects of the nursing staff, applying an instrument on knowledge and application of body mechanics in the care of patients, in the Hospital. Alfredo Noboa Montenegro, Ecuador, from July to
\end{abstract}

\section{Resumen}

Introducción. El ejercicio de la enfermería hospitalaria requiere una alta demanda de esfuerzo físico, que por desconocimiento puede originar lesiones musculoesqueléticas. Objetivo. Proponer un plan educativo en mecánica corporal para el personal de enfermería hospitalaria. Metodología. Con base a una investigación previa de tipo descriptiva, prospectiva, transversal, con diseño no experimental, en una muestra de 124 sujetos del personal de enfermería, aplicándoles un instrumento sobre conocimientos y aplicación de la mecánica corporal en el cuidado de

\footnotetext{
${ }^{1}$ Universidad Estatal de Bolívar, Facultad Ciencias de la Salud y del Ser Humano, Carrera de Enfermería, Guaranda, Ecuador.gnaranjo@ueb.edu.ec' https://orcid.org/0000-0003-1786-9979

2 Universidad del Zulia, Venezuela, Facultad de Medicina, lilianarojasg17@gmail.com, https://orcid.org/0000-0003-2714-8649

${ }^{3}$ Universidad Estatal de Bolívar, Facultad Ciencias de la Salud y del Ser Humano, Carrera de Enfermería, Guaranda, Ecuador. molalla@ueb.edu.ec' https://orcid.org/0000-0002-8358-9273
} 
December 2018. Results. The theoretical and practical principles of body mechanics in the practice of hospital nursing, can and should be achieved through training activities. Conclusion. The educational program allows the updating of knowledge, prevention of musculoskeletal injuries in nursing personnel through an adequate application of body mechanics, promoting the occupational health of those who practice this profession. pacientes, en el Hospital Alfredo Noboa Montenegro, Ecuador, desde julio a diciembre de 2018. Resultados. Los principios teóricos y prácticos de la mecánica corporal en el ejercicio de la enfermería hospitalaria, puede y debe lograrse a través de actividades de capacitación. Conclusión. El programa educativo permite la actualización de conocimientos, prevención de lesiones musculoesqueléticas en el personal de enfermería mediante una aplicación adecuada de la mecánica corporal, promocionando la salud laboral de quienes ejercen esta profesión.

Palabras clave: mecánica corporal, lesiones musculoesqueléticas, plan

educativo, enfermería.
Keywords: body mechanics, musculoskeletal injuries, educational plan, nursing.

\section{Introducción}

El diseño y aplicación de planes educativos se ha extendido a todas las áreas del saber (Menor et al, 2017), de hecho, la formación del conocimiento y el perfeccionamiento de un arte o destreza, parte de la docencia estructurada o no (Jordán et al., 2011), desde quienes ostentan el conocimiento hacia los que lo requieren, para desenvolverse apropiadamente en su medio habitual.

En el campo de la salud humana, se han implementado planes educativos para la prevención, curación y rehabilitación de la población. El Ministerio de Salud Pública en Ecuador (2019), estableció los lineamientos específicos para tal fin, los cuales han recibido el nombre de manual de educación y comunicación para la promoción de la salud. Afortunadamente dichas iniciativas se han extendido a los trabajadores, quienes también son susceptibles a enfermar.

Dentro del personal de salud, el profesional de enfermería comparte funciones de líder, atención ambulatoria, hospitalaria o comunitaria (De Arco y Suarez, 2018), lo cual representa un esfuerzo mayor en el desempeño de sus responsabilidades. De todas estas actividades, una de las más exigentes desde el punto de vista físico es la enfermería hospitalaria, motivo por el cual, diversas investigaciones han reportado las lesiones musculoesqueléticas, como una complicación causante de absentismos en esta población laboral Harari, (2011); Zanzii, (2020), Olalla et al. (2020), llegando a ser la mayor causa de incapacidad a nivel mundial (Organización Mundial de la Salud [OMS], 2020). 
Estos trastornos musculoesqueléticos son variados y sus síntomas difieren en cuanto a su ubicación y frecuencia, reportándose lumbalgia, cervicalgia, dolor en rodillas y afección del sistema osteomioarticular en general (Rosario \& Amézquita, 2014; Duque, et al., 2011; Harari, 2010), lo cual ocurre muy probablemente por desconocimiento de la mecánica corporal, durante la aplicación de técnicas y procedimientos la atención, manipulación y movilización de los pacientes (Kozier et al., 2005).

Para prevenir los trastornos musculoesqueléticos, el personal de enfermería debe conocer los principios y la aplicación correcta de la mecánica corporal (Naranjo, et al., 2021), por ello es necesario la intervención a través de la educación con programas de prevención sobre estos desórdenes, con el objetivo de proporcionar conocimientos que conlleven a reducir su ocurrencia. Todo esto beneficiará a las instituciones u organizaciones (ambientes saludables, disminución del absentismo laboral e incapacidad medica por estas causas) y al personal para evitar la aparición de estas enfermedades.

La intervención educativa implica un proceso de planificación de acciones, orientadas a promover un cambio de conducta en términos de conocimientos, actitudes o prácticas (Jordán et al., 2011), en tal sentido, la presente investigación tuvo como objetivo proponer un plan educativo sobre mecánica corporal, partiendo del establecimiento del conocimiento actual, en enfermeras hospitalarias.

\section{Metodología}

Se realizó una investigación descriptiva, prospectiva, transversal, con diseño no experimental (Hernández, et al., 2014; Arias, 2006), ejecutándose una evaluación diagnóstica en una muestra intencional de 124 miembros del personal de enfermería (86 profesionales y 38 auxiliares), a quienes se les aplicó un instrumento sobre conocimientos y aplicación de la mecánica corporal en el cuidado de pacientes, en el Hospital Alfredo Noboa Montenegro del cantón Guaranda, Ecuador, desde julio a diciembre de 2018.

Como criterio de inclusión se tomaron: ser miembro del personal del hospital ámbito del estudio, con antigüedad laboral no menor de un año, aceptación voluntaria para formar parte de la investigación, sin antecedentes de trastornos musculoesqueléticos, no embarazadas en los últimos dos años. Se excluyó al personal que no acepto participar y quienes tuvieron antecedentes patológicos. A toda participante de la investigación se le informó el objetivo de la misma y consintieron por escrito su deseo de participar, apegado a los principios de ética en el trabajo con humanos (World Medical Association, 2013).

Los instrumentos utilizados previa validación fueron: a) cuestionario para determinar el nivel de conocimiento sobre la mecánica corporal (Arone et al.,2016), y b) cuestionario sobre la aplicación de los principios de la mecánica corporal (Frontado \& Rodríguez, 2015). El 
tratamiento estadístico se realizó bajo los parámetros de la estadística descriptiva, calculando valores absolutos, relativos, promedios con desviación estándar y su significancia estadística.

\section{Resultados}

Toda propuesta de un plan educativo parte del diagnóstico de la situación problema de estudio (Betancourt et al., 2021). En la presente investigación se procedió a determinar el nivel de conocimiento y aplicación de los principios de la mecánica corporal, en el desempeño del personal de enfermería, al atender pacientes en el Hospital Alfredo Noboa Montenegro del cantón Guaranda, Ecuador. En tal sentido, se obtuvieron los siguientes resultados: grado deficiente de conocimiento en el 78,23\% (54,84\% grado bajo y $23,39 \%$ muy bajo) y un uso inadecuado de los principios de la mecánica corporal en el 77,42\%, siendo mayor de manera significativa $(p<0,001)$ en las enfermeras profesionales $(81,25 \%)$ que en las auxiliares $(18,75 \%)$, con una asociación significativa $(\mathrm{p}<0,01)$ entre esto dos parámetros, independientemente del área hospitalaria donde se encuentre asignado personal.

Con respecto a la severidad de los síntomas musculoesqueléticos referido por el personal de enfermería, se evidencio que los tres primeros lugares fueron catalogados moderados y localizados en hombro $(65,85 \%)$, cuello $(65,85 \%)$ y rodilla $(64,58 \%)$ y leves en codo/antebrazo $(72,09 \%)$, cadera/pierna $(71,79 \%)$ y dorso/lumbar $(68,85 \%)$; mientras que los severos fueron los menos frecuentes y se presentaron mayormente en rodillas $(10,42 \%)$, hombros $(9,76 \%)$ y región dorso/lumbar $(8,19 \%)$. Cabe mencionar que en forma general los síntomas musculoesqueléticos están presentes en todas las regiones corporales, siendo leves un $63.63 \%$ y moderados en un 36.6\%. Para evitar estas lesiones es necesario conocer y aplicar los principios de la mecánica corporal, que describen la forma correcta de utilizar el sistema musculoesquelético (Pincay et al., 2021).

A partir de los resultados mostrados anteriormente, se procedió a elaborar una propuesta de un plan educativo, bajo los principios docentes modernos en andragogía, contemplando los contenidos teóricos básicos sobre mecánica corporal, adaptados al tipo de trabajo del personal de enfermería hospitalario, sin minimizar la importancia de los aspectos procedimentales y actitudinales.

Siguiendo los lineamientos de Brito et al., (2021) y Cisneros (2021), el plan educativo propuesto tiene un primer componente teórico virtual, ejecutado a través de una plataforma amigable, la cual permite la interacción en tiempo real y diferido, entre el facilitador y los cursantes, de tal manera que, en todo momento, se aclaran las dudas que puedan surgir. El segundo componente es práctico-presencial, para ejecutar lo aprendido en forma teórica. La carga es de 20 horas académicas, impartido en dos horas diarias, por facilitadores expertos en cada tema, evidenciado por el currículo vitae de los mismos. Las estrategias educativas se muestran en la tabla 1 . 


\section{Tabla 1}

Estrategias educativas del plan educativo en mecánica corporal para el personal de enfermería hospitalaria

\begin{tabular}{|c|c|c|}
\hline Estrategia & Objetivo & Resultado esperado \\
\hline $\begin{array}{l}\text { Formación sobre mecánica } \\
\text { corporal. }\end{array}$ & $\begin{array}{l}\text { Fortalecer el conocimiento } \\
\text { sobre mecánica corporal. }\end{array}$ & $\begin{array}{l}\text { El personal de enfermería identifica los } \\
\text { factores de riesgo para lesiones } \\
\text { musculoesqueléticas. }\end{array}$ \\
\hline $\begin{array}{l}\text { Promoción de la prevención } \\
\text { de lesiones por mala } \\
\text { aplicación de la mecánica } \\
\text { corporal. }\end{array}$ & $\begin{array}{l}\text { Ejercitar en forma } \\
\text { adecuada la mecánica } \\
\text { corporal. }\end{array}$ & $\begin{array}{l}\text { Aplicar en forma adecuada la mecánica } \\
\text { corporal. }\end{array}$ \\
\hline $\begin{array}{l}\text { Prevención de las } \\
\text { complicaciones } \\
\text { musculoesqueléticas. }\end{array}$ & $\begin{array}{l}\text { Identificar las } \\
\text { manifestaciones tempranas } \\
\text { de las lesiones } \\
\text { musculoesqueléticas. }\end{array}$ & $\begin{array}{l}\text { Aplicar adecuada y oportunamente los } \\
\text { cuestionarios que detectan lesiones } \\
\text { musculoesqueléticas. }\end{array}$ \\
\hline $\begin{array}{l}\text { Evaluación del nivel de } \\
\text { conocimiento sobre la } \\
\text { mecánica corporal. }\end{array}$ & $\begin{array}{l}\text { Evaluar el conocimiento } \\
\text { teórico-práctico bridado en } \\
\text { el curso de formación } \\
\text { sobre mecánica corporal. }\end{array}$ & $\begin{array}{l}\text { Dominar contenidos teóricos y adquisición de } \\
\text { destrezas en mecánica corporal. }\end{array}$ \\
\hline
\end{tabular}

Se inicia el curso con el envío por correo electrónico del contenido del mismo, como se muestra en la tabla 2, especificando su objetivo académico, contenidos, evaluaciones y cronograma.

\section{Tabla 2}

\section{Contenido del plan educativo en mecánica corporal para el personal de enfermería hospitalaria}

Objetivo académico: dominar contenidos teóricos y destrezas en mecánica corporal en el personal de enfermería hospitalaria.

\begin{tabular}{ll}
\hline Mecánica corporal & $\begin{array}{l}\text { Definición, importancia y normas. } \\
\text { Elementos de la mecánica corporal }\end{array}$ \\
$\begin{array}{l}\text { Estabilidad } \\
\text { Trabajo físico }\end{array}$ & $\begin{array}{l}\text { Traslado de pacientes. } \\
\text { Levantamiento de objetos, adopción de posición del } \\
\text { pie y sentada, traslado de objetos, transporte de } \\
\text { pacientes. } \\
\text { Definición, causas, factores de riesgo, evaluación y } \\
\text { prevención. }\end{array}$ \\
Descripción de los trastornos musculoesqueléticos & $\begin{array}{l}\text { Características, ubicación, frecuencia y severidad. } \\
\text { Tipos, importancia, cuestionario de kuorinka. }\end{array}$ \\
Instrumentos para medir SME & Aplicación de los principios de la mecánica corporal. \\
Descripción de las posturas asumidas en enfermería. & Conocer y aplicar la mecánica corporal, test \\
Prevención de los trastornos musculoesqueléticos. & Kourinca. \\
Mecánica corporal, trastornos musculoesqueléticos y & SME.
\end{tabular}


Las dos primeras sesiones son teóricas, donde su diserta sobre los conceptos y principios de la mecánica corporal y luego tres encuentros prácticos en grupos no mayores de 25 personas por facilitador, en un ambiente hospitalario o que lo represente, de tal forma que se puedan mostrar los movimientos, posturas, técnicas y alcances de una buena mecánica postural, en el ejercicio de la enfermería hospitalaria. La evaluación es realizada de común acuerdo entre el facilitador y los cursantes, pero siempre contempla la evidencia de adquisición de habilidades y destrezas en la mecánica corporal en el desempeño de la enfermería hospitalaria. Finalmente se entrega un certificado de la formación sobre mecánica postural en enfermería hospitalaria, emitido por la unidad de salud donde se realiza la capacitación.

En conclusión, el conocimiento y ejecución de los principios teóricos y prácticos de la mecánica corporal en el ejercicio de la enfermería hospitalaria, puede y debe lograrse a través de actividades de capacitación. Diversas investigaciones de Ríos (2018) y Zanzii (2020), muestran que son efectivas en la reducción de ausencias al trabajo por trastornos musculoesqueléticos y en tal sentido, todas las unidades sanitarias que cuenten con servicios de hospitalización, deben vigilar por la salud de sus trabajadores en enfermería (OMS, 2020), instruyéndoles y adiestrándoles en tan importante área de la salud ocupacional, como lo es la mecánica corporal durante la jornada laboral.

\section{Conclusiones}

El programa educativo fortalece el conocimiento del personal de enfermería en mecánica corporal permitiendo adquirir eficacia, efectividad para el desarrollo de movimientos al realizar el cuidado a los pacientes o para el transporte de equipos o insumos médicos durante su jornada laboral.

El programa educativo permite llevar a cabo la prevención de lesiones musculoesqueléticas en el personal de enfermería mediante una aplicación adecuada de la mecánica corporal promocionando la salud laboral de quienes ejercen esta profesión.

El programa educativo ayuda a detectar de manera temprana el desarrollo de complicaciones musculoesqueléticas en el personal de enfermería, mediante la valoración de cuestionarios diseñados para riesgo laboral.

El programa educativo contribuye a la evaluación de la aplicación de la mecánica corporal en el personal de enfermería que está laborando en el área hospitalaria, así como también al personal de nuevo ingreso, permitiendo corregir o detectar oportunamente enfermedades propias de su actividad laboral. 


\section{Referencias Bibliográficas}

Arias, F. (2006). El proyecto de investigación: Introducción a la metodología científica. (Episteme (ed.); 6ta. ed.).

Arone, L., Becerra, G., Jorge, C. \& Zamalloa, K. (2016). Conocimiento y aplicación de la mecánica corporal de la enfermera de centro quirúrgico de un hospital de Lima. Tesis de grado, Universidad Cayetano Heredia de Perú. https://repositorio.upch.edu.pe/

Betancourt, I., López, A., Furones, J., Castro, M. \& Lima, L. (2021). Intervención educativa para el mejoramiento humano desde la cultura en los estudiantes de ciencias médicas. EDUMECENTRO, 13(2), 108-127. http://scielo.sld.cu/scielo.php?script=sci_arttext\&pid=S2077$28742021000200108 \& \operatorname{lng}=\mathrm{es} \& \operatorname{lng}=\mathrm{es}$

Brito, S., Basualto, L. \& Reyes, L. (2021). La metodología de proyecto social en la educación superior: una oportunidad para armonizar el proyecto de vida con el proyecto de sociedad. Educación, 30(58), 107 127. https://dx.doi.org/10.18800/educacion.202101.005

Cisneros, J. (2021). Mecánica corporal del personal de salud en la Unidad de Cuidados Intensivos, Hospital Luis Gabriel Dávila. Trabajo de grado, Universidad Regional Autónoma de los Andes. Ecuador. https://dspace.uniandes.edu.ec/handle/123456789/12255

De Arco, O. \& Suarez, Z. (2018). Rol de los profesionales de enfermería en el sistema de salud colombiano. Univ. Salud. 20(2),171-182. http://dx.doi.org/10.22267/rus.182002.121.

Duque, I., Zuluaga D. \& Pinilla, A. (2011). Prevalencia de lumbalgia y factores de riesgo en enfermeros y auxiliares en la ciudad de Manizales. Promoción de la Salud. 16(1): 27-38. http://www.scielo.org.co/pdf/hpsal/v16n1/v16n1a02.pdf

Frontado, K. \& Rodríguez, M. (2015). Uso de la mecánica corporal en enfermeras del servicio de emergencia del Hospital Belén de Trujillo. Trabajo de grado, Universidad Privada Antenor Orrego, Perú. https://repositorio.upao.edu.pe/handle/20.500.12759/1694

Harari, F. (2010). Trastornos músculo-esqueléticos en auxiliares de enfermería de un Hospital en Quito. Rev EIDOS. 3: 32-45. https://revistas.ute.edu.ec/index.php/eidos/article/view/68

Hernández, R., Fernández, C. \& Baptista, P. (2014). Metodología de la Investigación. (Mc Graw Hill Interamericana (ed.); 6ta ed.).

Jordán, M., Pachón, L., Blanco, M. \& Achiong, M. (2011). Elementos a tener en cuenta para realizar un diseño de intervención educativa. Rev Méd Electrón. 
33(4), 540-546. http://scielo.sld.cu/scielo.php?script=sci_arttext\&pid=S168418242011000400017

Kozier, B., Erb, G., Berman, A. \& Snyder, S. (2005). Fundamentos de Enfermería. Concepto, proceso y práctica. (Interamericana (ed.)).

Menor, M., Aguilar, M., Mur, N. \& Santana,C. (2017). Efectividad de las intervenciones educativas para la atención de la salud. Revisión sistemática. Medisur. 15 (1),71-84.

Ministerio de Salud Pública del Ecuador. (2019). Educación y comunicación para la promoción de la salud. Manual. Subsecretaría Nacional de Promoción de la Salud e Igualdad. Dirección de Promoción de la Salud Quito. https://www.salud.gob.ec/wp-

content/uploads/2019/12/manual_de_educaci\%C3\%B3n_y_comunicaci\%C3 \%B3n_para_promoci\%C3\%B3n_de_la_salud0254090001575057231.pdf

Naranjo, G., Rojas, L. \& Olalla, M. (2021). Conocimiento y aplicación de la mecánica corporal: Enfermeras de un hospital de Ecuador. Pol. Con. 6(7), 295-312. http://polodelconocimiento.com/ojs/index.php/es

Olalla, M., Naranjo, G., López, S., Muñoz, M. \& Bayas, F. (2020). Body mechanics and complications in the nursing personnel of the emergency service of the Luis Vernaza General Hospital (Guayaquil-Ecuador). Electrón J. Gen Med. 17(2), em192. https://doi.org/10.29333/ejgm/7809

Organización Mundial de la Salud [OMS]. (2020). La Organización Mundial de la Salud y sus asociados hacen un llamamiento urgente para que se invierta en el personal de enfermería (Comunicado de prensa). https://www.who.int/es/news/item/07-04-2020-who-and-partners-call-forurgent-investment-in-nurses

Pincay, M., Chiriboga, G. \& Vega, V. (2021). Posturas inadecuadas y su incidencia en trastornos músculo esqueléticos. Revista de la Asociación Española de Especialistas en Medicina del Trabajo. 30(2), 161-168. http://scielo.isciii.es/scielo.php?script=sci_arttext\&pid=S1132$62552021000200161 \& \operatorname{lng}=\mathrm{es} \& \operatorname{lng}=\mathrm{es}$.

Ríos, M. (2018). Trastornos musculoesqueléticos del miembro superior en el Hospital Militar de Matanzas. Revista Médica Electrónica. 40(6), 1819-1834. http://scielo.sld.cu/scielo.php?script=sci_arttext\&pid=S1684$18242018000601819 \& \operatorname{lng}=\mathrm{es} \& \operatorname{lng}=\mathrm{es}$.

Rosario, R. \& Amézquita, T. (2014). Prevalencia de trastornos músculo-esqueléticos en el personal de esterilización en tres hospitales públicos. Med Segur Trab. 60

https://scielo.isciii.es/scielo.php?script=sci_abstract\&pid=S0465546X2014000100004 
Zanzii, J. (2020). Fundamentos teóricos de la mecánica corporal en la movilización de pacientes en el ámbito de enfermería. Mas Vita. Rev. Cienc. Salud. 2(1),815. https://doi.org/10.47606/ACVEN/MV0001

World Medical Association (2013). Declaración de Helsinki de la Asociación Médica Mundial. Principios éticos para las investigaciones médicas en seres humanos. 64th. WMA General Asambly, Fortaleza Brazil. https://www.wma.net/policies-post/wma-declaration-of-helsinki-ethicalprinciples-for-medical-research-involving-human-subjects/2013/.

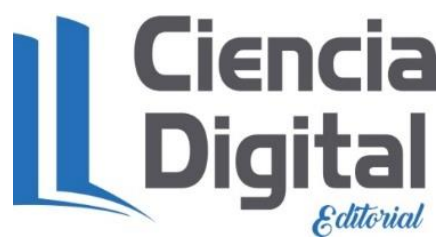




\section{PARA CITAR EL ARTÍCULO INDEXADO}

Naranjo Chávez, G. M., Rojas González, L. R., \& Olalla García, M. H. (2021). Propuesta de un plan educativo en mecánica corporal para el personal de enfermería hospitalaria . Anatomía Digital, 4(4), 112-121. https://doi.org/10.33262/anatomiadigital.v4i4.1920

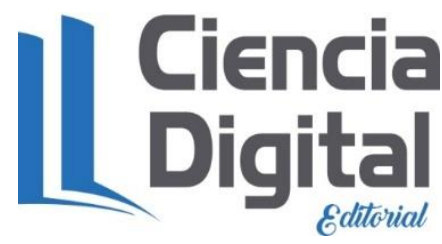

El artículo que se publica es de exclusiva responsabilidad de los autores y no necesariamente reflejan el pensamiento de la Revista Anatomía Digital.

El artículo queda en propiedad de la revista y, por tanto, su publicación parcial y/o total en otro medio tiene que ser autorizado por el director de la Revista Anatomía Digital.
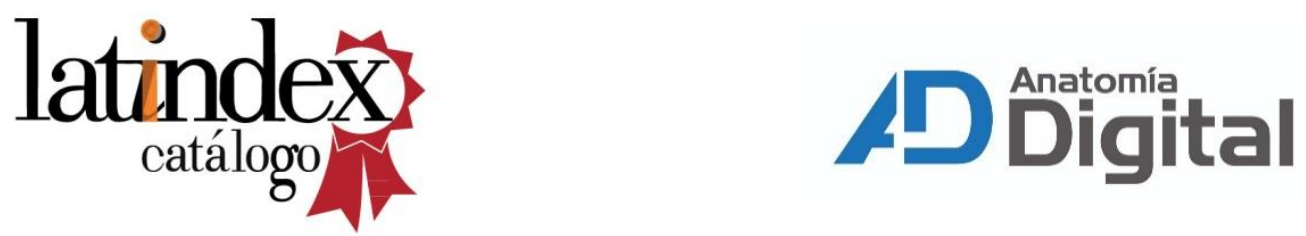